\title{
Fatores associados à imagem corporal em estudantes de Educação Física
}

\author{
Tatiana Rodrigues da Silva ${ }^{1}$ \\ Guilherme Saenger ${ }^{1}$ \\ Érico Felden Pereira ${ }^{2}$ \\ ${ }^{1}$ Universidade Federal de Santa Maria, Santa Maria, RS, Brasil \\ ${ }^{2}$ Universidade do Estado de Santa Catarina, Florianópolis, SC, Brasil
}

\begin{abstract}
Resumo: Objetivou-se investigar alguns fatores associados à percepção da imagem corporal em acadêmicos de educação física. 230 estudantes responderam a um questionário com questões relacionadas à imagem corporal e práticas de atividade física. Avaliações antropométricas foram realizadas. Análises ajustadas da insatisfação pelo excesso de peso e pela magreza foram apresentadas. As prevalências de insatisfação com a imagem corporal foram de $62,8 \%$ para o sexo masculino e de $67 \%$ para o feminino. A insatisfação pela magreza foi 2,71 (IC95\%: 1,47-4,99) vezes maior no grupo masculino e a insatisfação pelo excesso de peso foi 2,22 (IC95\%: 1,40-3,54) vezes maior no grupo feminino. Devido às altas prevalências de insatisfação com a imagem corporal identificadas, sugere-se que os cursos de formação dêem maior destaque a temas como imagem corporal e padrões de beleza nas atividades curriculares, possibilitando uma melhor atuação junto a grupos com distorções de imagem corporal, comuns em escolas e academias.
\end{abstract}

Palavras-chave: Imagem Corporal. Estado Nutricional. Sexo. Estudantes.

\section{Factors associated with body image in Physical Education students}

\begin{abstract}
The aim of this study was to investigate factors associated with body image in physical education students. 230 students answered a questionnaire with body image and physical activities habits questions. Anthropometrics evaluations were taken. Adjusted analyses with underweight and overweight dissatisfactions were performed. $62.8 \%$ of males and $67 \%$ of females showed body dissatisfaction. The dissatisfaction because they regarded themselves as being too thin was 2.71 (Cl95\%: 1.47-4.99) fold higher in males and the dissatisfaction because they perceived themselves as overweight was 2.22 (C195\%: 1.403.54) fold higher in females. Due to high body dissatisfaction prevalence identified, we suggest the higher attention about body image and beauty standards questions in curricular activities. This would enable the better professional performance with body dissatisfaction and distorted body image groups common in schools and academies.
\end{abstract}

Key Words: Body image. Nutritional status. Gender. Sex. Students.

\section{Introdução}

O padrão de beleza, enquanto conceito culturalmente construído pelo imaginário social modifica-se em diferentes contextos históricos e sociais, mas, apesar disso, o culto ao corpo, de uma forma ou de outra, sempre esteve associado à imagem de poder, beleza e mobilidade social (ANDRADE; BOSI, 2003; PEREIRA et al., 2009). Conforme discutem Cash e Pruzinski (1990) a imagem corporal pode ser interpretada como a forma que o corpo se apresenta para os indivíduos, tanto com relação a eles mesmos, quanto aos outros indivíduos do seu meio. Tratase de um construto multifatorial ligado, tanto a fatores neurofisiológicos e anatômicos, como sociais e culturais.

A literatura atual (CONTI et al., 2005; MALDONADO, 2006; BAUER; KIRCHENGAST,
2006; GRAUP et al., 2008; PEREIRA et al., 2009; PEREIRA et al., 2008) destaca a crescente influência e pressão exercidas pela mídia no sentido da homogeneização de corpos pelos padrões de beleza em voga levando os indivíduos, especialmente adolescentes e mulheres, a uma maior insatisfação com seu corpo.

Coqueiro et al. (2008) sistematizaram os fatores que podem estar associados à satisfação com a imagem corporal considerando as seguintes dimensões: percepção da expectativa dos pais e amigos para ser mais magro, localização geográfica da residência (grandes centros urbanos ou cidades menores), depressão e comportamentos de saúde (auto-estima, comportamento alimentar, uso de esteróides e dependência de exercício, percepção equivocada 
do peso corporal, idade, razões pessoais para querer perder peso (aparência, saúde ou estética).

Neste contexto, alguns grupos parecem apresentar peculiaridades com relação à imagem e satisfação corporal, como é o caso de acadêmicos de educação física. Secchi et al. (2009), em um estudo realizado com acadêmicas de educação física, destaca que, apesar dessas apresentarem uma auto-percepção corporal e um Índice de Massa Corporal (IMC) considerados normais, encontravam-se descontentes com sua aparência física.

Resultados semelhantes foram apresentados no estudo de Bosi et al. (2008) também com acadêmicas de educação física. $O$ grupo analisado, apesar de apresentar uma média geral de IMC adequada, em grande parte estava insatisfeito com sua imagem corporal. Aquelas acadêmicas com índices de sentimento de desvalorização em relação ao corpo classificados como moderados/graves corresponderam a $30 \%$ do grupo que gostaria de diminuir mais de $2 \mathrm{~kg}$. Além disso, é importante destacar também o observado no estudo de Marcondelli et al. (2008), com universitários da área da saúde, incluindo acadêmicos de educação física, que mostrou um elevado percentual de acadêmicos com hábitos alimentares inadequados.

Assim, os estudantes universitários e, em especial aqueles dos cursos de educação física, merecem uma particular atenção com relação ao tema imagem corporal. Ao escolherem esta profissão os acadêmicos vêem-se associados a uma imagem de corpos esculturais e hábitos alimentares saudáveis o que pode levar a índices ainda maiores de insatisfação. Porém, o excesso de carga extracurricular, devido à abrangência da área, verificado neste grupo, dentre outros fatores, acaba por favorecer a adoção de hábitos não saudáveis no decorrer da formação.

Destaca-se, por fim, que se trata de profissionais que estarão atuando diretamente com públicos com elevados índices de insatisfação e de distorção da imagem corporal enquanto educadores em escolas e academias, 0 que torna a discussão sobre o tema, neste grupo, especialmente relevante. Assim, analisou-se alguns fatores associados à insatisfação com a imagem corporal em um grupo de acadêmicos de educação física, considerando os anos de formação do curso de graduação.

\section{Método}

\section{População e Amostra}

Para a formação da amostra foi primeiramente realizado um levantamento junto ao Departamento de Registro e Controle Acadêmico para identificar o número total de alunos regulamente matriculados no curso de educação física. Após está identificação utilizou-se a proposta de Rodrigues (2002) para obter-se o número mínimo de acadêmicos para que a amostra fosse representativa do curso. A amostra então foi estratificada por ano de graduação e por habilitação (licenciatura e bacharelado). A amostra final do estudo foi formada por 230 acadêmicos dos cursos de educação física licenciatura e bacharelado, de uma instituição federal de ensino do sul do Brasil, sendo 129 homens com média de idade de $21,54(3,25)$ anos e 101 mulheres com média de idade 20,85 $(2,49)$ anos.

\section{Procedimentos Gerais}

Primeiramente o projeto foi aprovado pelo Comitê de Ética em Pesquisa da instituição de origem sob protocolo número 0273.0.243.000-10 e seguiu as normas da Resolução 196/96 do Conselho Nacional de Saúde sobre pesquisas envolvendo seres humanos. A amostra foi selecionada aleatoriamente e por conglomerado, sendo que todos os acadêmicos presentes em sala de aula no momento das coletas foram convidados e aceitaram fazer parte do estudo. Os acadêmicos, após receberem informações sobre os objetivos do estudo e procedimentos para coleta de dados, assinaram o Termo de Consentimento Livre e Esclarecido.

\section{Instrumentos}

Para investigar a percepção da imagem corporal foi aplicado o instrumento proposto por Stunkard et al. (1983) composto por um continuum de nove silhuetas. A partir deste conjunto de silhuetas foram realizadas duas perguntas aos acadêmicos: Qual o número da silhueta que você considera mais semelhante com sua aparência real? E Qual o número da silhueta que você gostaria de ter? Para verificar a percepção da imagem corporal foi realizada uma subtração entre a silhueta corporal atual e a silhueta corporal ideal; se essa variação fosse igual a zero, os acadêmicos foram classificados como satisfeitos e se diferente de zero, como insatisfeitos. Uma diferença positiva indica 
insatisfação pelo excesso de peso (desejo de diminuir o tamanho da silhueta) e diferença negativa, uma insatisfação pela magreza (desejo de aumentar a silhueta).

Para verificar-se o IMC dos acadêmicos utilizou-se de medidas antropométricas (massa corpórea e estatura) e, para investigar o nível de atividade física, foi aplicado o Questionário Internacional de Atividade Física (IPAQ- versão curta), de acordo com Matsudo et al. (2002), que contém perguntas a respeito do tempo que o indivíduo teria gastado fazendo atividades físicas moderadas e vigorosas na última semana. A classificação do nível de atividade foi realizada pela duração e pela frequência da atividade durante a semana. Desta forma, os acadêmicos foram classificados como ativos, se tivessem 300 minutos ou mais de atividades, ou sedentários, se tivessem menos que 300 minutos de atividades.

\section{Análise Estatística}

Foram realizadas análises descritivas (média, desvio padrão e frequência). Como a maior parte das variáveis não apresentou distribuição normal, considerando o resultado do teste de KolmogorovSmirnov, optou-se pela análise dos dados por meio de testes não paramétricos. Foi utilizado o teste de Kruskal-Wallis para verificar a diferença entre médias, a correlação de Spearman para verificar associações entre o estado nutricional, percepção da imagem corporal e o nível de atividade física e o Qui-quadrado para verificar a diferença entre percentuais para variáveis categóricas. A análise de regressão de Poisson permitiu estimar as razões de prevalências para insatisfação pelo excesso de peso e pela magreza, de acordo com a idade, sexo, IMC, atividade física e ano de curso. Foram analisados modelos não ajustados e ajustados, considerando para o modelo ajustado aquelas variáveis que apresentaram $\mathrm{p}<0,25$ no teste do Qui-quadrado (HOSMER; LENESHOW, 1989). Em todas as análises considerou-se como nível de probabilidade de significância o $p<0,05$.

\section{Resultados}

As características descritivas da amostra investigada de acordo com o sexo foram apresentadas na Tabela 1. Foram encontradas diferenças estatisticamente significativas nas seguintes variáveis: silhueta ideal, insatisfação pelo excesso de peso e pela magreza e na prevalência de sobrepeso.

Tabela 1. Dados descritivos a diferenças entre os sexos.

\begin{tabular}{lccc}
\hline \multicolumn{1}{c}{ Variáveis $^{\text {a }}$} & Masculino & Feminino & p-valor $^{\mathbf{b}}$ \\
\hline Idade (anos) & $21,54(3,25)$ & $20,85(2,49)$ & 0,104 \\
Estatura (cm) & $1,78(0,06)$ & $1,64(0,07)$ & 0,001 \\
Massa Corporal (Kg) & $75,61(10,29)$ & $59,89(9,71)$ & 0,001 \\
IMC (kg/m ${ }^{2}$ S & $23,84(2,65)$ & $22,09(3,12)$ & 0,001 \\
Silhueta Real & $3,99(1,11)$ & $4,03(3,78)$ & 0,057 \\
Silhueta Ideal & $4,07(0,71)$ & $3,47(3,54)$ & 0,001 \\
Imagem Corporal (\%) & & & \\
$\quad$ Satisfeito & 37,2 & 33,0 & 0,516 \\
$\quad$ Insatisfação pelo excesso de peso & 28,9 & 53,0 & 0,001 \\
$\quad$ Insatisfação pela magreza & 33,9 & 14,0 & 0,001 \\
IMC (\%) & & & \\
$\quad$ Normal & 70,3 & 85,0 & 0,009 \\
$\quad$ Sobrepeso & 26,6 & 11,0 & 0,003 \\
$\quad$ Obeso & 3,1 & 4,0 & 0,722 \\
IPAQ (\%) & & & \\
$\quad$ Ativos & 72,1 & 66,0 & 0,329 \\
\hline
\end{tabular}

${ }^{a}$ variáveis expressão em média (desvio padrão) e em percentual

b-valor do teste de Kruskal-Wallis para dados contínuos e do Qui-quadrado para dados categóricos

Como ilustrado na Figura 1, analisou-se a prevalência de sobrepeso/obesidade dos acadêmicos de acordo com o ano de graduação. Os achados indicaram que apenas no terceiro ano do curso de graduação houve diferença significativa entre os sexos $(p=0,001)$. 
Figura 1. Prevalência de sobrepeso/obesidade dos acadêmicos de acordo com o ano de graduação).

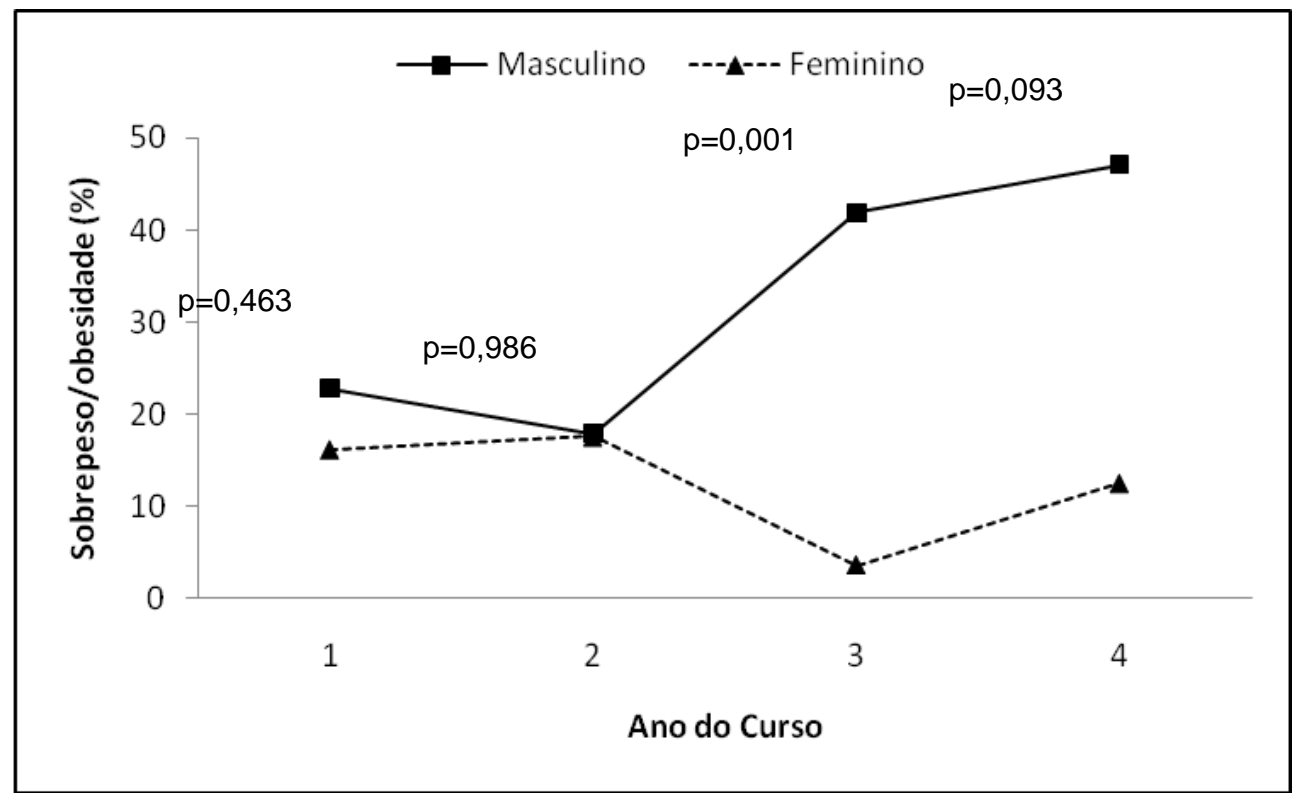

Na Figura 2, foram apresentadas as prevalências de insatisfação pela magreza dos acadêmicos de acordo com o ano de graduação. Os resultados apontaram uma diferença significativa entre os sexos no terceiro ano do curso de graduação $(p=0,001)$. Enquanto no sexo masculino as prevalências de insatisfação pela magreza foram semelhantes em todos os semestres; no sexo feminino verificou-se uma importante redução no final do curso, não sendo identificadas acadêmicas com insatisfação pela magreza (Figura 2).

Considerando a insatisfação pelo excesso de peso, identificou-se um aumento da insatisfação no decorrer do curso para ambos os sexos (Figura 3), sendo observadas diferenças significativas entre moças e rapazes no terceiro ano $(\mathrm{p}=0,001)$.

Figura 2. Prevalência de insatisfação com a magreza de acordo com o ano do curso de graduação.

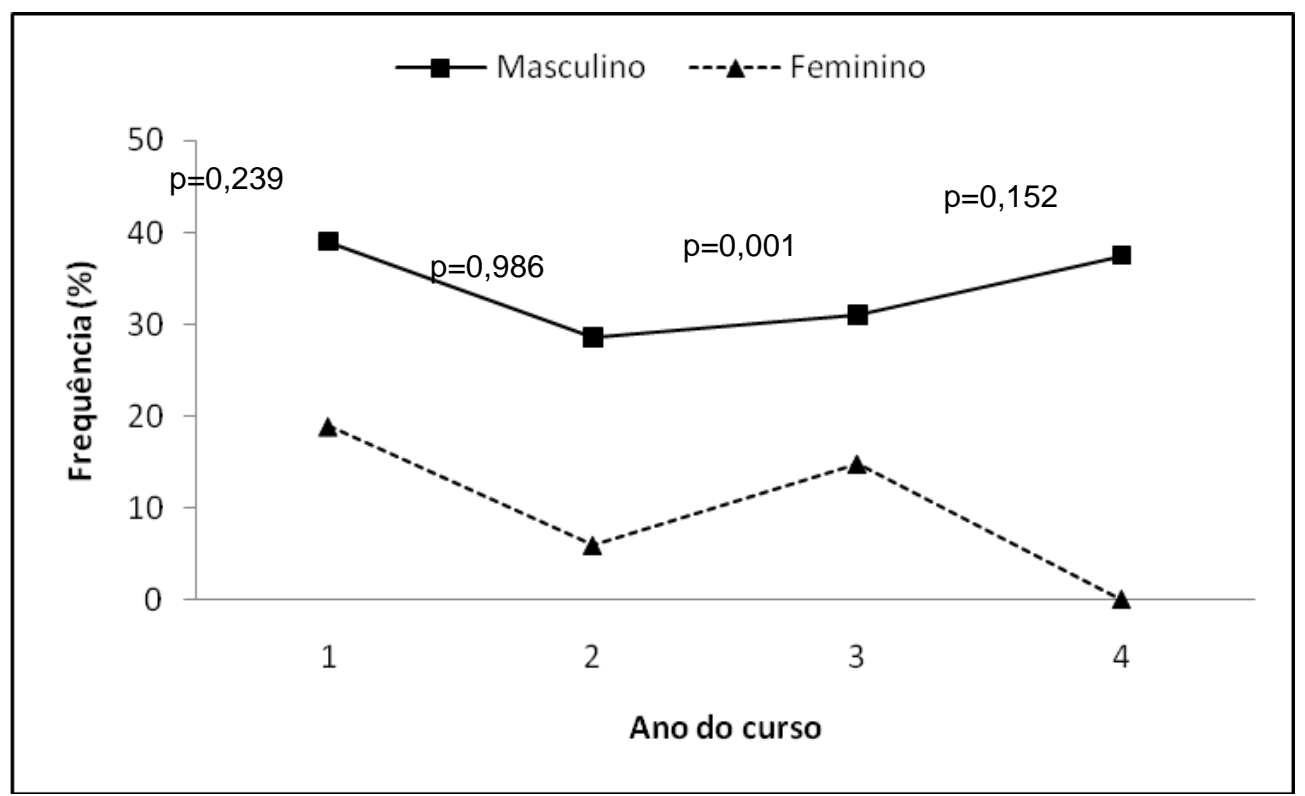


Figura 3. Prevalência de insatisfação pelo excesso de peso de acordo com o ano do curso de graduação.

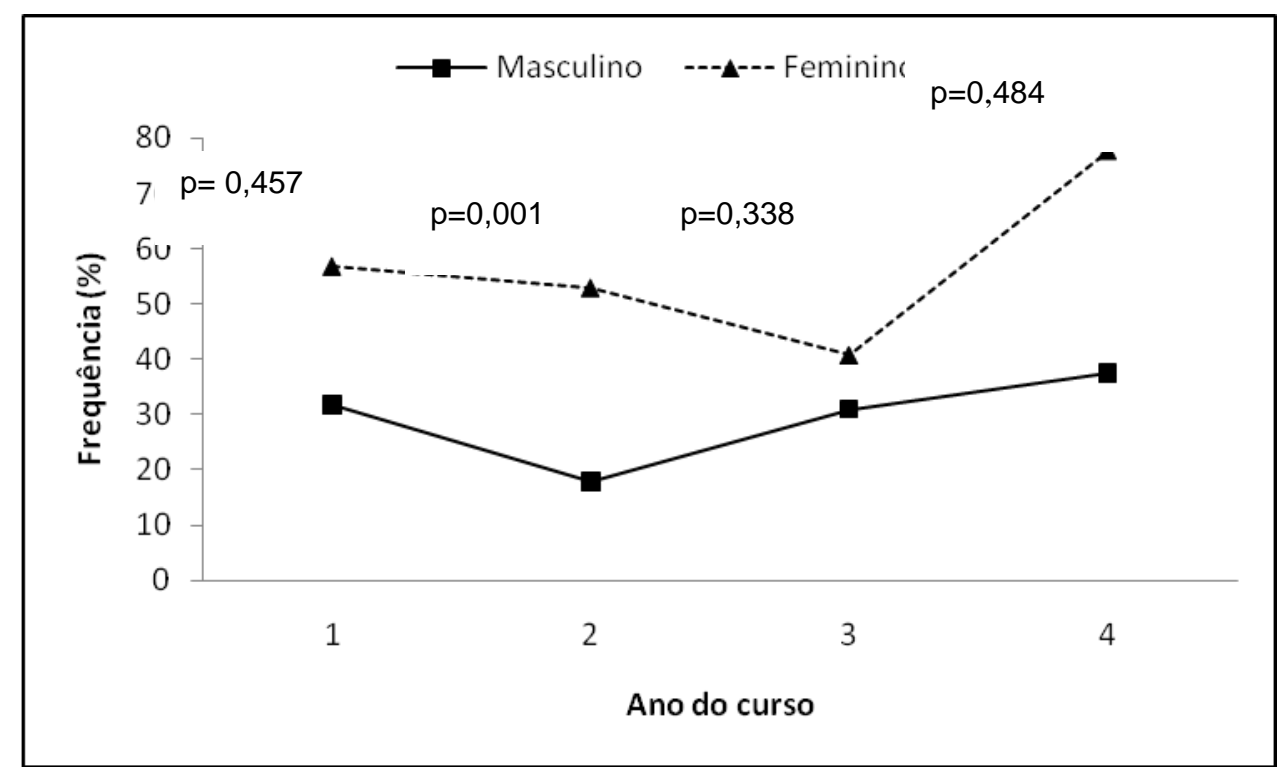

Tabela 2. Razões de prevalências (RP) utilizando-se como variável dependente a insatisfação pela magreza.

\begin{tabular}{|c|c|c|c|c|c|}
\hline \multirow{2}{*}{ Variáveis } & \multirow{2}{*}{ Prevalência (\%) } & \multicolumn{2}{|c|}{ Análise não ajustada ${ }^{a}$} & \multicolumn{2}{|c|}{ Análise ajustada ${ }^{b}$} \\
\hline & & RP (IC95\%) & $\mathbf{p}$ & RP (IC95\%) & $\mathbf{p}$ \\
\hline $\begin{array}{l}\text { Idade (anos) } \\
17-18 \\
19-20 \\
21-22 \\
23-24 \\
25-38 \\
\end{array}$ & $\begin{array}{l}23,1 \\
28,8 \\
33,3 \\
16,2 \\
11,5\end{array}$ & $\begin{array}{l}2,01(0,60-6,71) \\
2,50(0,80-7,81) \\
2,90(0,94-8,90) \\
1,41(0,38-5,13) \\
1\end{array}$ & 0,384 & Excluído & - \\
\hline $\begin{array}{l}\text { Sexo } \\
\text { Masculino } \\
\text { Feminino } \\
\end{array}$ & $\begin{array}{l}33,9 \\
14,0\end{array}$ & $\begin{array}{c}2,42(1,40-4,18) \\
1 \\
\end{array}$ & 0,002 & $\begin{array}{c}2,71(1,47-4,99) \\
1\end{array}$ & 0,001 \\
\hline $\begin{array}{l}\text { IMC } \\
\text { Eutróficos } \\
\text { Sobrepeso/ obesidade }\end{array}$ & $\begin{array}{c}29,8 \\
7,8\end{array}$ & $\begin{array}{c}3,82(1,44-10,02) \\
1\end{array}$ & 0,007 & $\begin{array}{c}4,51(1,62-12,54) \\
1\end{array}$ & 0,004 \\
\hline $\begin{array}{l}\text { Atividade física } \\
\text { Ativos } \\
\text { Insuficientemente ativos }\end{array}$ & $\begin{array}{l}28,3 \\
21,0 \\
\end{array}$ & $\begin{array}{c}1,35(0,78-2,34) \\
1\end{array}$ & 0,286 & Excluído & - \\
\hline $\begin{array}{l}\text { Ano do curso } \\
1 \\
2 \\
3 \\
4 \\
\end{array}$ & $\begin{array}{l}29,5 \\
20,0 \\
23,2 \\
24,0\end{array}$ & $\begin{array}{c}1,47(0,74-2,91) \\
1 \\
1,16(0,54-2,47) \\
1,20(0,48-2,99)\end{array}$ & 0,894 & Excluído & - \\
\hline
\end{tabular}

$\mathrm{Na}$ Tabela 2 foram apresentados os cálculos das razões de prevalência considerando a insatisfação pela magreza como variável dependente. Os resultados da análise não ajustada revelaram associação significativa considerando as variáveis sexo e IMC. A prevalência de insatisfação pela magreza foi 2,71 vezes maior no sexo masculino na análise ajustada. A maior parte dos acadêmicos insatisfeitos pela magreza foi classificada como eutrófica $(29,8 \%)$ e, na análise considerando o estado nutricional, foi observada maior insatisfação pela magreza nestes acadêmicos (4,51 vezes maior do que os acadêmicos classificados com sobrepeso/obesidade). 
Tabela 3. Razões de prevalências (RP) utilizando-se como variável dependente a insatisfação pelo excesso de peso.

\begin{tabular}{|c|c|c|c|c|c|}
\hline \multirow{2}{*}{ Variáveis } & \multirow{2}{*}{ Prevalência (\%) } & \multicolumn{2}{|c|}{ Análise não ajustada ${ }^{a}$} & \multicolumn{2}{|c|}{ Análise ajustada $^{\mathrm{b}}$} \\
\hline & & RP (IC95\%) & $\mathbf{p}$ & RP (IC95\%) & $\mathbf{p}$ \\
\hline $\begin{array}{l}\text { Idade (anos) } \\
17-18 \\
19-20 \\
21-22 \\
23-24 \\
25-38\end{array}$ & $\begin{array}{l}46,2 \\
33,9 \\
38,3 \\
45,9 \\
38,5\end{array}$ & $\begin{array}{c}1,36(0,83-2,23) \\
1 \\
1,13(0,62-1,08) \\
1,35(0,82-2,23) \\
1,13(0,70-1,82)\end{array}$ & 0,447 & Excluído & - \\
\hline $\begin{array}{l}\text { Sexo } \\
\text { Masculino } \\
\text { Feminino } \\
\end{array}$ & $\begin{array}{l}28,9 \\
53,0\end{array}$ & $\begin{array}{c}1 \\
1,83(1,31-2,56)\end{array}$ & $<0,001$ & $\begin{array}{c}1 \\
2,22(1,40-3,54)\end{array}$ & 0,001 \\
\hline $\begin{array}{l}\text { IMC } \\
\text { Eutróficos } \\
\text { Sobrepeso/ obesidade }\end{array}$ & $\begin{array}{l}66,7 \\
31,5\end{array}$ & $\begin{array}{c}2,12(1,57-2,84) \\
1\end{array}$ & $<0,001$ & $\begin{array}{c}2,43(1,51-3,91) \\
1\end{array}$ & $<0,001$ \\
\hline $\begin{array}{l}\text { Atividade física } \\
\text { Ativos } \\
\text { Insuficientemente ativos }\end{array}$ & $\begin{array}{l}37,2 \\
41,9\end{array}$ & $\begin{array}{c}1 \\
1,13(0,78-1,62) \\
\end{array}$ & 0,520 & Excluído & - \\
\hline $\begin{array}{l}\text { Ano do curso } \\
1 \\
2 \\
3 \\
4 \\
\end{array}$ & $\begin{array}{l}43,6 \\
31,1 \\
35,7 \\
52,0\end{array}$ & $\begin{array}{c}1,41(0,85-2,32) \\
1 \\
1,15(0,65-2,01) \\
1,67(0,94-2,97)\end{array}$ & 0,232 & $\begin{array}{c}1,29(0,69-2,41) \\
1 \\
1,06(0,53-2,10) \\
1,53(0,71-3,34)\end{array}$ & 0,502 \\
\hline
\end{tabular}

Os cálculos das razões de prevalência, considerando a insatisfação pelo excesso de peso como variável dependente, foram apresentados na Tabela 3. Os resultados da análise não ajustada e ajustada também revelaram associações significativas para as variáveis sexo e IMC. A prevalência de insatisfação pelo excesso de peso foi 1,83 vezes maior no sexo feminino e na análise ajustada este valor foi 2,22 vezes maior. Igualmente ao verificado na análise multivariada, considerando a insatisfação pela magreza, os acadêmicos eutróficos também apresentaram maior insatisfação pelo excesso de peso.

Apesar de grande parte das acadêmicas insatisfeitas pelo excesso de peso ser classificada como eutrófica $(66,7 \%)$ observou-se uma prevalência de insatisfação pelo excesso de peso de 2,12 vezes maior nestas acadêmicas (análise não ajustada) e de 2,43 vezes maior na análise ajustada. Em suma, apesar das acadêmicas estarem mais insatisfeitas pelo excesso de peso e os acadêmicos pela magreza, em ambos os casos os indivíduos eutróficos apresentaram maior prevalência de insatisfação com a imagem corporal.
A idade, o ano do curso de graduação e o nível de atividade física não foram associados, tanto com a insatisfação pela magreza, como pelo excesso de peso.

\section{Discussão}

O estudo apresentado mostrou algumas importantes análises na compreensão da percepção da imagem corporal em acadêmicos de educação física. Confirmou-se a grande prevalência de insatisfação com a imagem corporal; um aumento do sobrepeso durante o curso de graduação, especialmente no sexo masculino; a não diminuição da insatisfação, principalmente pelo excesso de peso, no decorrer do curso e a ausência de associação entre insatisfação com a imagem corporal com o ano do curso e com o nível de atividade física em uma amostra com grande quantidade de atividade física semanal. Além disso, confirmou-se a importância de se avaliar, tanto a insatisfação pela magreza, como pelo excesso de peso, considerando diferenças importantes entre moças e rapazes.

Os achados do presente estudo apontaram que $(62,8 \%)$ dos universitários e $(67 \%)$ das universitárias apresentaram insatisfação com sua 
imagem corporal, sendo que o sexo feminino encontrou-se mais insatisfeito pelo excesso de peso e o masculino pela magreza. Estes resultados estão de acordo com as prevalências encontradas em pesquisas com universitários (QUADROS et al., 2010; THOMAS et al., 2010; GONCCALVES et al., 2008; COQUEIRO et al.,

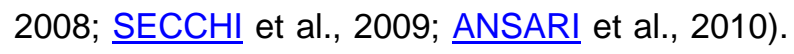
Destaca-se que uma percepção aumentada do peso corporal pode estar significativamente associada a maiores risco de sintomas depressivos, especialmente nas mulheres, como observado em estudo recente com universitários norte-americanos (HARRING et al., 2010).

Embora se trate de um estudo transversal buscou-se analisar os dados de acadêmicos de todos os anos da graduação, discutindo possíveis modificações nas freqüências de insatisfação com a imagem corporal e de estado nutricional. Uma tendência de aumento do sobrepeso foi observada, especialmente no grupo masculino, acompanhada por um possível aumento da insatisfação pelo excesso de peso o que remete à necessidade de um maior acompanhamento da saúde física e emocional dos acadêmicos durante o curso.

Apesar disso, é importante destacar que esta análise sofre influências das mudanças sociais que decorreram durante o período de graduação e os alunos dos últimos anos não podem ser considerados uma sequência linear dos primeiros. Desta forma, tanto o período de graduação pode ter levado a essas diferenças, corroborando com tendências observadas por Bosi et al. (2008), como as maiores influências socioculturais relacionados aos padrões de beleza e hábitos alimentares podem ter contribuído para esses resultados, o que deve ser mais bem explorado em análises longitudinais.

Segundo Russo (2005) a insatisfação dos universitários com a sua imagem corporal é um fenômeno que atinge ambos os sexos, já que todos sofrem influências, especialmente da mídia. Apesar disso, tem-se identificado que a insatisfação de homens e mulheres é diferenciada, sendo importante a análise da insatisfação, tanto pelo excesso de peso, como pela magreza, traduzida, provavelmente, pelo desejo de mais massa muscular nos homens.

A maioria dos universitários foi classificada como eutrófica e, mesmo no grupo com estado nutricional normal, observou-se uma grande insatisfação com a imagem corporal, corroborando com outros estudos apresentados pela literatura (BOSI et al., 2006; QUADROS et al., 2010; MIKOLAJCZYK et al., 2010; LAUS et al., 2008; ALVARENGA et al., 2010). Conforme destacam Pereira et al. (2009) e Quadros et al. (2010) essas divergências, entre o estado nutricional e a insatisfação com a imagem corporal, possivelmente, estão relacionadas a uma preocupação exagerada com a estética corporal culturalmente imposta, fazendo com que o padrão de beleza idealizado não corresponda aos padrões considerados adequados para a saúde, o que poderia ser trabalho por meio de medidas de educação em saúde.

A associação entre a imagem corporal e o estado nutricional e ausência de associação com o nível de atividade física em acadêmicos de educação física confirma um recente levantamento dessas variáveis em acadêmicos de educação física em acadêmicos de uma instituição estadual do Paraná ( $\underline{\mathrm{RECH}}$ et al., 2010). Considera-se relevante, neste aspecto, apontar que possíveis medidas de intervenção focando na saúde global de estudantes universitários, com base somente na atividade física, podem não apresentar resultados satisfatórios para um melhor conhecimento a aceitação de sua imagem corporal e que medidas mais globais são necessárias.

Os futuros profissionais de educação física estarão atuando diretamente com pessoas com insatisfação com a imagem corporal e com distúrbios de imagem corporal e alimentares e, desta forma, a identificação correta da melhor forma de intervenção pode ser promovida, mesmo que subjetivamente, por meio de uma melhor compreensão de sua própria imagem corporal e dos padrões de beleza impostos socialmente, já que esta auto-percepção também se forma no contato interpessoal. Destaca-se, neste contexto, a análise publicada por Lüdorf (2009) na qual foi discutido que, apesar dos 
temas sobre estética e corpo estarem sendo abordados, de alguma forma, durante a formação de em educação física, tal discussão deveria estar mais presente e melhor sistematizada devido a sua relevância na atuação profissional.

As principais limitações apontadas neste estudo relacionam-se ao desenho de coleta do tipo transversal, no qual conclusões sobre o comportamento das variáveis analisadas no decorrer dos anos do curso de graduação precisam ser realizadas com cuidado. Além da diminuição natural de alunos nos últimos anos do curso $o$ que pode influenciar nas análises estatísticas. Assim, um acompanhamento de tais variáveis, desde a entrada dos acadêmicos até a conclusão do curso, bem como intervenções educativas, para um melhor conhecimento de questões relacionadas à imagem corporal, são recomendadas.

\section{Conclusão}

Prevalências altas de insatisfação com a imagem corporal nos acadêmicos eutróficos foram verificadas. As universitárias apresentaramse mais insatisfeitas pelo excesso de peso e os universitários apresentaram-se mais insatisfeitos pela magreza, possivelmente por um desejo de mais massa muscular. O alto nível de atividade física identificado nos acadêmicos não esteve associação às variáveis de imagem corporal analisadas, ao contrário do estado nutricional.

As prevalências de insatisfação com a imagem corporal não diminuíram no decorrer da formação acadêmica e houve uma tendência de aumento das prevalências de sobrepeso e obesidade, especialmente no sexo masculino. Estudos longitudinais durante a formação do profissional de educação física com relação à imagem corporal são recomendados e um destaque maior ao tema durante a graduação é sugerido.

\section{Referências}

ALVARENGA, M. S.; PHILIPPI, S. T.; LOURENÇO, B. H.; SATO, P. M.; SCAGLIUSI, F. B. Insatisfação com a imagem corporal em universitárias brasileiras. Jornal Brasileiro de Psiquiatria, Rio de Janeiro, v.59. n.1, p.4451, 2010. Disponível em:
$<$ http://www.scielo.br/pdf/jbpsiq/v59n1/v59n1a07.p df>. Acesso em: 15 de Abril de 2010.

ANDRADE, A.; BOSI, M. L. M. Mídia e subjetividade: impacto no comportamento alimentar feminino. Revista de Nutrição, Campinas, v.16, n.1, p. 117-125, 2003. http://dx.doi.org/10.1590/S1415$\underline{52732003000100012}$

ANSARI, W. E.; CLAUSEN, S. V.; MABHALA, A.; STOCK, C. How do I look? Body image perceptions among university students from England and Denmark. International Journal of Environmental Research and Public Health, v.7, n.2, p. 583-595, 2010. Disponível em: <http://www.mdpi.com/1660-4601/7/2/583/pdf>. Acesso em: 16 de Abril de 2010.

BAUER, M.; KIRCHENGAST S. Body composition, weight status, body image and weight control practices among female adolescents from eastern Austria.

Anthropologischer Anzeiger, v. 64, n.3, p.32131, 2006. Disponível em: $<$ http://www.ncbi.nlm.nih.gov/pubmed/17128935>. Acesso em: 13 de Março de 2010.

BOSI, M. L. M.; RONIR RAGGIO LUIZ, R. R.; MORGADO, C. M. C.; COSTA, M. L. S.; CARVALHO, R. J. Autopercepção da imagem corporal entre estudantes de nutrição: um estudo no município do Rio de Janeiro. Jornal Brasileiro de Psiquiatria, Rio de Janeiro, v.55, n.2, p. 108113, 2006. Disponível em:

$<$ http://www.scielo.br/pdf/jbpsiq/v55n2/v55n2a03.p df>. Acesso em: 21 de Março de 2010.

BOSI, M. L. M.; LUIZ, R. R.; UCHIMURA, K. Y.; OLIVEIRA, F. P. Comportamento alimentar e imagem corporal entre estudantes de educação física. Jornal Brasileiro de Psiquiatria, Rio de Janeiro, n. 57, n. 1, p. 28-33, 2008. http://dx.doi.org/10.1590/S0047$\underline{20852008000100006}$

CASH, T. F.; PRUZINSKY, T. Body images: development, deviance and change. New York: The Guilford Press 1990.

CONTI, M. A.; FRUTUOSO, M. F. P.; GAMBARDELLA, A. M. D. Excesso de peso e insatisfação corporal em adolescentes. Revista de Nutrição, Campinas, v.18, n.4, p. 491-497, 2005. http://dx.doi.org/10.1590/S1415$\underline{52732005000400005 .}$

COQUEIRO, R. S.; PETROSKI, E. L.; PELEGRINI, A.; BARBOSA, A. R. Insatisfação com a imagem corporal: avaliação comparativa da associação com estado nutricional em universitários. Revista de Psiquiatria do Rio Grande do Sul, Porto Alegre, v.30, n.1, p.31-38, 
2008. http://dx.doi.org/10.1590/S010181082008000100009.

GONÇALVES, T. D.; BARBOSA, M. P.; ROSA, L. C. L.; RODRIGUES, A. M. Comportamento anoréxico e percepção corporal em universitários. Jornal Brasileiro de Psiquiatria, Rio de Janeiro, v.57, n.3, p.166-170, 2008. Disponível em:

$<$ http://www.scielo.br/pdf/ibpsiq/v57n3/02.pdf>. Acesso em: 05 de Maio de 2010.

GRAUP, S.; PEREIRA, E. P.; LOPES, A. S.; ARAÚJO, V. C.; LEGNANI, R. F. S.; BORGATTO, A. F. Associação entre a percepção da imagem corporal e indicadores antropométricos de escolares. Revista Brasileira de Educação Física e Esportes, São Paulo, v.22 n.2, 2008. Disponível em:

$<$ http://www.revistasusp.sibi.usp.br/pdf/rbefe/v22n 2/v22n2a4.pdf>. Acesso em: 05 de Maio de 2010.

HARRING, H.A.; MONTGOMERY, K.; HARDIN, J. Perceptions of body weight, weight management strategies, and depressive symptoms among US college students. Journal American College Health, v.59, n.1, p. 43-50, 2010. Disponível em: <http://www.ncbi.nlm.nih.gov/pubmed/20670928>. Acesso em: 06 de Maio de 2010.

HOSMER, D. W.; LENESHOW, S. Applied logistic regression. New York; Wiley, 1989.

LAUS, M. F.; MOREIRA, R. C. M.; COSTA, T. M. B. Diferenças na percepção da imagem corporal, no comportamento alimentar e no estado nutricional de universitárias das áreas de saúde e humanas. Revista de Psiquiatria do Rio Grande do Sul. Porto Alegre, v.31, n.3, p. 192-196, 2009. http://dx.doi.org/10.1590/S0101$\underline{81082009000300009}$.

LÜDORF, S. M. A. Corpo e formação de professores de educação física. Interface Comunicação, Saúde, Educação. Botucatu, v. 13, n.28, p. 99-110, 2009. Disponível em: $<$ http://www.scielo.br/pdf/icse/v13n28/v13n28a09. pdf>. Acesso em: 18 de Maio de 2010.

MALDONADO, G. R. A educação física e o adolescente: a imagem corporal e a estética da transformação na mídia impressa. Revista Mackenzie de Educação Física e Esporte, v.5, n.1, p. 59-76, 2006. Disponível em: $<$ http://www3.mackenzie.br/editora/index.php/rem ef/article/viewFile/1302/1006>. Acesso em: 23 de Maio de 2010.

MARCONDELLI, P.; COSTA, T. H. M.; SCHMITZ, B. A. S. Nível de atividade física e hábitos alimentares de universitários do $3^{\circ}$ ao $5^{\circ}$ semestres da área da saúde. Revista de Nutrição, Campinas, v.21, n.1, p. p. 39-47, 2008.
http://dx.doi.org/10.1590/S1415$\underline{52732008000100005}$

MATSUDO, S. M.; MATSUDO, V. R.; ARAUJO, T.; ANDRADE, D.; ANDRADE, E.; OLIVEIRA, L.; et al. Nível de atividade física da população do Estado de São Paulo: análise de acordo com o gênero, idade, nível socioeconômico, distribuição geográfica e de conhecimento. Revista Brasileira Ciência e Movimento. Brasília, v.10, n.4, P. 4150, 2002. Disponível em:

$<$ http://portalrevistas.ucb.br/index.php/RBCM/articl e/viewFile/469/495>. Acesso em: 11 de Maio de 2010.

MIKOLAJCZYK, R. T.; MAXWELL, A. E.; ANSARI, W. E.; STOCK, C.; PETKEVICIENE, J.; GUILLEN-GRIMA, F. Relationship between perceived body weight and body mass index based on self- reported height and weight among university students: a cross-sectional study in seven European countries. BMC Public Health, v.10, n. 40, 2010. Disponível em:

$<$ http://www.biomedcentral.com/1471-

2458/10/40>. Acesso em: 11de Maio de 2010.

PEREIRA, É. F.; GRAUP, S.; LOPES, A. S.; BORGATTO, A. F.; DARONCO, L. S. E. Percepção da imagem corporal de crianças e adolescentes com diferentes níveis sócioeconômicos na cidade de Florianópolis, Santa Catarina, Brasil. Revista Brasileira de Saúde Materno Infantil, Recife, v.9, n.3, p. 253-262, 2009. http://dx.doi.org/10.1590/S1519$\underline{38292009000300004}$

PEREIRA, É. F.; TEIXEIRA, C. S., BORGATTO, A. F.; DARONCO, L. S. E. Relação entre diferentes indicadores antropométricos e a percepção da imagem corporal em idosas ativas. Revista de Psiquiatria Clínica, São Paulo, v.36, n.2, p.54-9, 2008. http://dx.doi.org/10.1590/S0101$\underline{60832009000200003}$.

QUADROS, T. M. B.; GORDIA, A.P.; MARTINS, C. R.; SILVA, D. A. S.; FERRARI, E. P.; PETROSKI, E. L. Imagem corporal em universitários: associação com estado nutricional e sexo. Motriz, Rio Claro, v.16, n.1, p.78-85, 2010. http://dx.doi.org/10.5016/1980$\underline{6574.2010 \mathrm{v} 16 \mathrm{n} 1 \mathrm{p} 78}$

RECH, C. R.; ARAÚJO, E. D. S.; VANAT, J. R. Autopercepção da imagem corporal em estudantes do curso de educação física. Revista Brasileira de Educação Física e Esporte, São Paulo, v.24, n.2, p. 285-92, 2010. Disponível em: $<$ http://www.revistasusp.sibi.usp.br/pdf/rbefe/v24n 2/v24n2a11.pdf >. Acesso em: 24 de Maio de 2010.

RUSSO, R. Imagem corporal: construção através da cultura do belo. Movimento \& Percepção, 
Espírito Santo de Pinhal, v.5, n.6, p.80-90, 2005.

Disponível em:

$<$ http://www.portalsaudebrasil.com/artigospsb/psic 0029.pdf>. Acesso em: 04 de Abril de 2010.

SECCHI, K.; CAMARGO, B. V.; BERTOLDO, R. B. Percepção da imagem corporal e representações sociais do corpo. Psicologia:

Teoria e Pesquisa, Brasília, v.25, n.2, p. 229-236, 2009. http://dx.doi.org/10.1590/S0102-

$\underline{37722009000200011}$

STUNKARD, A.J.; SORENSEN, T.;

SCHULSINGER, F. Use of the Danish Adoption

Register for the study of obesity and thinness. In:

KETY, S.S.; ROWLAND, L.P.; SIDMAN, R.L.;

MATTHYSSE, S.W. (Ed.). The Genetics of

Neurological and Psychiatric Disorders. New

York: Raven Press, p. 115-120, 1983.

THOMAS, J.; KHAN, S.; ABDULRAHMAN, A. A.

Eating attitudes and body image concerns among female university students in the United Arab

Emirates. Appetite. v.54, n.3, p. 595-598, 2010.

Disponível em:

<http://www.ncbi.nlm.nih.gov/pubmed/20172004>.

Acesso em: 04 de Abril de 2010.

Endereço:

Tatiana Rodrigues da Silva

Rua Marques do Herval, 611, Apartamento 07

Santa Maria RS Brasil

E-mail: rthaty@hotmail.com

Recebido em: 31 de agosto de 2010.

Aceito em: 28 de abril de 2011.

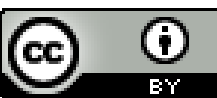

Motriz. Revista de Educação Física. UNESP, Rio Claro,

SP, Brasil - elSSN: 1980-6574 - está licenciada sob Creative Commons - Atribuicão 3.0 